\title{
The Association Between Blood Pressure and
}

\section{Headache in Postmenopausal Women: A Prospective Hospital-Based Study}

Liu Wang ${ }^{1, *}$

Hong-Ping Tan ${ }^{1} * *$

Rui Han ${ }^{2}$

'Department of Pain Management, Affiliated Hospital of North Sichuan Medical College, Nanchong, Sichuan Province, People's Republic of China; ${ }^{2}$ The Affiliated Yantai Yuhuangding Hospital of Medical College, Qingdao University, Yantai, Shandong Province, People's Republic of China

*These authors contributed equally to this work
Correspondence: Hong-Ping Tan Department of Pain Management, Affiliated Hospital of North Sichuan Medical College, I Maoyuan South Road, Shunqing District, Nanchong City, Sichuan Province, 637000, People's Republic of China

Tel +86-08I7226273I

Email tanhpdoctor@outlook.com
Background: Headache is the most frequent condition for outpatient patients because of neurological problems, but little is known about predisposing and enabling factors for headache patients.

Aim: To investigate the association between blood pressure (BP) and headache in postmenopausal women.

Methods: The postmenopausal women who were admitted to our hospital from January 2015 to December 2019 were screening according to the criteria. Their systolic BP (SBP), diastolic BP (DBP), pulse pressure (PP) were assessed and the information of age, body mass index, smoking status, caffeine consumption, cholesterol levels, and daily alcohol use were collected. Multiple logistic regression model was established to evaluate the association between BP and headache.

Results: A total of 1571 postmenopausal women were included in the analysis, including 953 headache-free population and 618 headache participants during the studied periods. We found that increasing SBP and PP were associated with the lower occurrence of migraine, tension-type headache (TTH), probable migraine, and unclassified headache $(\mathrm{P}<0.05)$. However, there was a negative association between DBP levels and the new occurrence of overall headache, but we did not find any relations of DBP with any subtypes of headache. Conclusion: There were negative associations of SBP and PP with new occurrence of headache, especially migraine and TTH, but there is no relationship between DBP and the subsequent development of headache.

Keywords: blood pressure, hormone, menopause, headache

\section{Introduction}

Headache is the most frequent condition for outpatient patients because of neurological problems. ${ }^{1}$ As a major public-health problem, recurrent or persistent headache has seriously negative impacts on personal qualities of life and socioeconomic burden. ${ }^{2,3}$ The commonest types of headache are migraine and tension-type headache (TTH), which belong to primary headaches. ${ }^{4}$ However, little is known about predisposing and enabling factors for headache patients.

Studies have shown that blood pressure (BP) has an association with headache, although researches on such association are still conflicting. It is commonly accepted that headache has a link with multiple diseases which causes abrupt and paroxysmal increase in BP in acute conditions. ${ }^{5}$ And the elevation of BP commonly appears in headache patients in the emergency department, which was 
significantly more than patients with other problems. ${ }^{6}$ Nevertheless, some studies suggested that the elevation of both systolic BP and pulse pressure may decrease headache prevalence through modulation of the baroreflex arch. ${ }^{7}$ And there is a research did not found an association between the change of BP and the occurrence of headache on patients with mild hypertension. ${ }^{8}$ Moreover, studies have shown that headache was a prevalent problem in postmenopausal women, and they experience an improvement in the frequency of headache at menopause..$^{9,10}$ However, studies focused on the association between blood pressure and headache in postmenopausal women were absent, and the specific definition of headache-free population is scarce in current research.

Thus, we conducted a hospital-based study to evaluate the possible relationship between BP and headache in postmenopausal women, especially migraine and TTH. Our study could provide a more convinced evidence by adjusting for multiple potential confounders and giving a uniform and specific definition of headache-free population.

\section{Methods}

\section{Subjects}

First of all, we found the information of all hospitalized patients in our hospital from January 2015 to December 2019 after approving by the Ethics Committee of Affiliated Hospital of North Sichuan Medical College, and this study was conducted in accordance with the Declaration of Helsinki. The participants who enrolled in this study were carefully selected from these hospitalized patients according to the inclusion criteria and exclusion criteria.

The inclusion criteria were like following: (1) Participants who are post-menopausal females; (2) Participants who had complete clinical information; (3) Participants who are willing to sign written informed consent and comply with the study; (4) Participants have not taken any hypertensive medications or accepted hormone replacement therapy (HRT) and headache prophylactic therapies.

The exclusion criteria include the following: (1) Participants are males or females who still have menstruation; (2) Participants with incomplete medical records; (3) Participants who refused to participate in this study; (4) Patients who were taking antihypertensive medications or receiving hormone replacement therapy.
All participants were asked by this question during follow-up: "Have you suffered from headache during the last month?". If the answer is 'Yes', the participants were classified as headache patients. Those who answered "no" were considered as the headache-free populations. All participants will be followed up at 1-month intervals through telephone and outpatient follow-up review, with a total follow-up of 2 years. Their BPs and cholesterol levels were measured, and their smoking status, physical activities, caffeine and alcohol consumptions were asked and recorded during every follow-up review.

\section{Headache Diagnoses}

During the follow-up reviews, the patients who developed headache were required to go to our hospital to receive examinations. The participants who had headache symptoms were categorized as: migraine group, probable migraine group, TTH group or unclassified headache group. And those migraine and TTH patients were diagnosed by physicians according to the criteria of International Classification of Headache Disorders, 3rd edition (beta version). ${ }^{11}$ Patients who have probable migraine means that they fulfill all but one of the diagnostic criteria for a subtype of migraine headache. ${ }^{12}$ Besides, the unclassified headache was defined by a headache which did not fulfill the characteristics of any diagnoses.

\section{Blood Pressure Assessment}

$\mathrm{Bp}$, including systolic BP (SBP), diastolic BP (DBP), pulse pressure (PP) and were measured by a digital sphygmomanometer. Participants must have a rest for at least 5 min before measuring their BP. The final recording data were calculated as the average systolic and diastolic pressures based on 3 seated resting measurements. The average BPs of all follow-up reviews were used in the final analysis. And the values of SBP were divided into 4 groups: $<120,120-139,140-159$ and $\geq 160 \mathrm{mmHg}$. Moreover, the values of DBP were divided into 4 groups as well: $<80,80-89,90-99$ and $\geq 100 \mathrm{mmHg}$. And the values of $P P$ were divided into $<45,45-54,55-64$ and $\geq 65$ $\mathrm{mmHg}$.

\section{Statistical Analysis}

The normally distributed data were presented as means \pm standard errors. The frequency and percentage (\%) were used for categorical variables. Multiple logistic regression model was established to evaluate the association between 
BP and headache. The BP categories were included in the model as continuous variables, thereby investigating a potential linear tendency and calculating the odd ration (OR) per $10 \mathrm{mmHg}$. This model also used by adjusting for age, body mass index (BMI), the status of current smoking and drinking alcohol, the levels of caffeine consumption and cholesterol. Statistical analysis was performed using SPSS, Version 22.0 (SPSS Inc., Chicago, IL).

\section{Results}

Figure 1 shows the diagram of screening process. In total, we collected the information of 11,259 hospitalized headache-free patients in our hospital from January 2015 to December 2019. A total of 9688 participants were excluded, including 7712 male patients or female patients who are not postmenopausal, 753 patients who refused to participate in this study, 661 participants who were receiving HRT and headache prophylactic therapies, and 562 patients who were taking antihypertensive medication. And there were 1571 postmenopausal women enrolled in this final study.
Table 1 shows the characteristics of the participants. The average age of these postmenopausal populations were 57.8 years, and their SBP and DBP were $138.5 \pm$ 16.9 (mean \pm SD) and $80.9 \pm 11.3 \mathrm{mmHg}$, separately. And the pulse pressure was $57.6 \pm 12.5 \mathrm{mmHg}$. The BMI of them were $24.4 \pm 3.6 \mathrm{~kg} / \mathrm{m}^{2}$, and the average cholesterol levels was $5.6 \mathrm{mmol} / \mathrm{L}$. Moreover, there were $24.5 \%$ participants who had daily smoking habits, and $7.5 \%$ participants who had daily alcohol use. Besides, $14.8 \%$ participants did activities that make them breathe hard at least 2 hours every day. Moreover, they also took caffeine and the daily average consumption was $103.7 \mathrm{mg}$.

Among these participants, 953 individuals said "No" to the question "Have you suffered from headache during the last month?" at each follow-up. And 618 participants had headache during the studied periods, including 125 patients suffered from migraine, 137 patients suffered from TTH, 155 patients had probable migraine, and 201 patients with unclassified headache

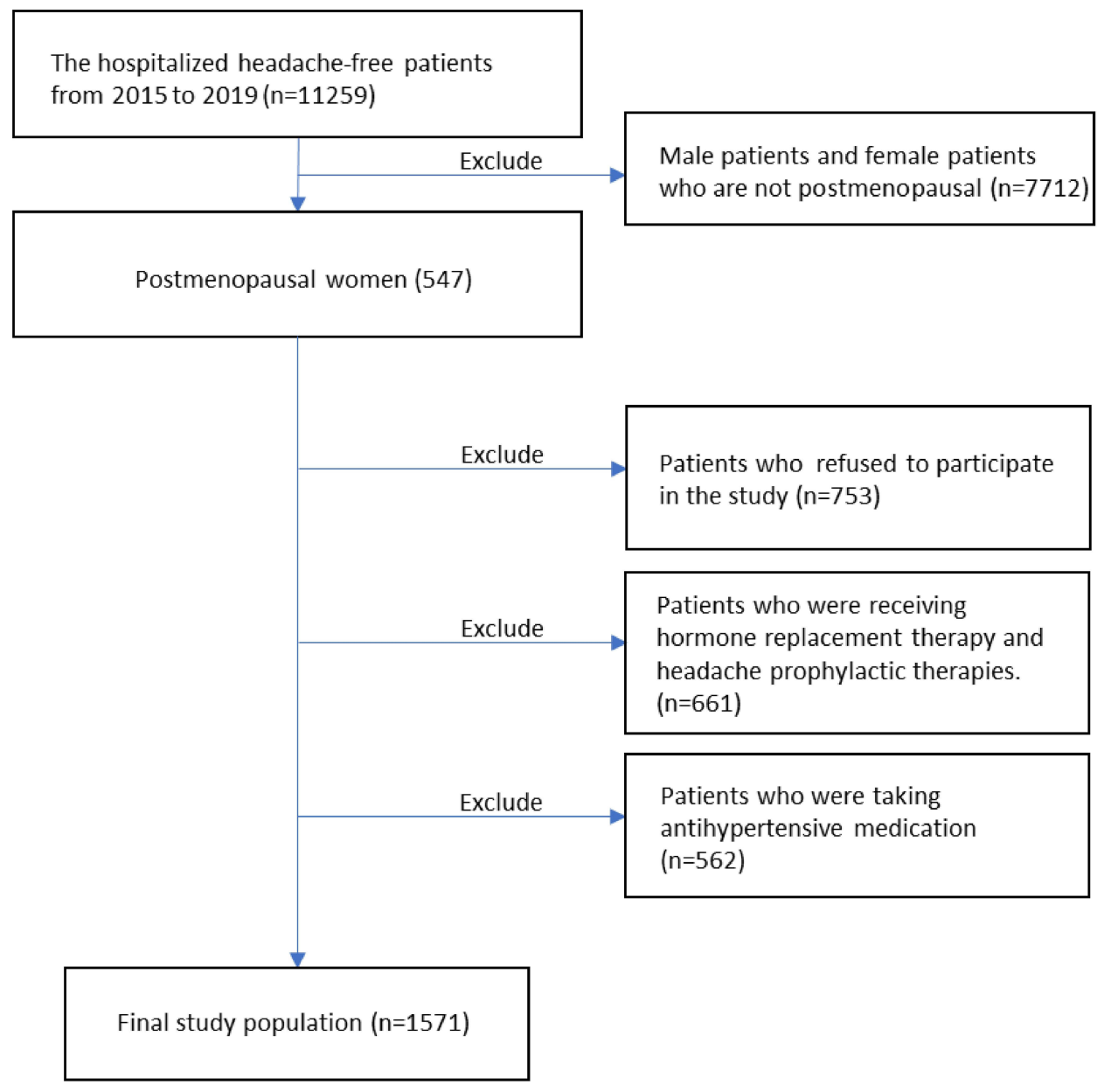

Figure I Flow diagram of participant recruitment. 
Table I Characteristics of the Participants

\begin{tabular}{|l|c|}
\hline Variables & Study Population $(\mathbf{n = 1 5 7 I )}$ \\
\hline Age, years & $57.8 \pm 14.5$ \\
Systolic blood pressure, $\mathrm{mmHg}$ & $138.5 \pm 16.9$ \\
Diastolic blood pressure, $\mathrm{mmHg}$ & $80.9 \pm 1 \mathrm{I} .3$ \\
Pulse pressure, $\mathrm{mmHg}$ & $57.6 \pm 12.5$ \\
Body mass index, $\mathrm{kg} / \mathrm{m}^{2}$ & $24.4 \pm 3.6$ \\
Current daily smokers & $385(24.5 \%)$ \\
Hard physical activity & $233(14.8 \%)$ \\
Caffeine consumption per day, $\mathrm{mg}$ & $103.7 \pm 71.8$ \\
Cholesterol levels, mmol/L & $5.6 \pm 1.3$ \\
Daily alcohol use & $118(7.5 \%)$ \\
\hline
\end{tabular}

(Table 2). Our multiple logistic model adjusting for the confounding factors were conducted and found that SBP and PP were negatively associated with the occurrence of any new headache $(\mathrm{P}<0.05)$. And increasing SBP and PP were associated with the lower occurrence of migraine, TTH, probable migraine, and unclassified headache $(\mathrm{P}<0.05)$. However, there was a negative association between DBP levels and the new occurrence of overall headache, but we did not find any relations of DBP with migraine, TTH, probable migraine, or unclassified headache.

\section{Discussion}

In this prospective study, we found a negative association of the new occurrence of headache, especially migraine and TTH, with SBP and PP. However, the DBP did not show any relationship with subsequent development of headache. Hopefully, this study could provide epidemiologic evidence for the pathological mechanism of headache.

Many studies have shown that elevated BP has an association with headache because primary headache is frequently occurred in hypertension. ${ }^{5,13}$ And some studies took the view that subjects with headache always had the risks of hypertension. ${ }^{14,15}$ Our results seem to be inconsistent with those studies, which mainly because that these cross-sectional studies cannot demonstrate causality. Our study found the negative relationship between headache and SBP and PP, which was consistent with several studies. For instance, a longitudinal study enrolled 10,366 men and 11,171 women have found that patients with migraine had lower SBP, and the elevated SBP could decrease the probability of migraine for $19 \%$ males and $25 \%$ females. $^{16}$

In this study, we enrolled 4 types of headaches into the model. Migraine and TTH were the two most common type of

Table 2 The Relationship Between Blood Pressure and Headache by Multiple Logistic Regression

\begin{tabular}{|c|c|c|c|c|c|}
\hline & Any Headache & Migraine & Tension Type Headache & Probable Migraine & Unclassified Headache \\
\hline Individuals (n) & 618 & 125 & 137 & 155 & 201 \\
\hline \multicolumn{6}{|l|}{ Systolic pressure, $\mathrm{mmHg}$} \\
\hline$<120$ & 1.00 (reference) & 1.00 (reference) & 1.00 (reference) & 1.00 (reference) & 1.00 (reference) \\
\hline $120-139$ & $0.85(0.73-1.11)$ & $0.78(0.61-0.93)$ & $0.91(0.78-1.05)$ & $0.85(0.61-1.09)$ & $1.14(0.78-1.56)$ \\
\hline $140-159$ & $0.72(0.61-0.90)$ & $0.65(0.43-0.88)$ & $0.83(0.61-0.92)$ & $0.86(0.66-1.08)$ & $0.65(0.45-0.92)$ \\
\hline$>160$ & $0.73(0.61-0.82)$ & $0.49(0.25-0.79)$ & $0.71(0.53-0.77)$ & $0.58(0.34-0.79)$ & $0.35(0.19-0.68)$ \\
\hline PER $10 \mathrm{mmHg}$ & $0.76(0.60-0.89)$ & $0.85(0.61-0.97)$ & $0.84(0.59-0.99)$ & $0.83(0.67-0.99)$ & $0.79(0.58-0.92)$ \\
\hline$P$ for linear association & $<0.001$ & $<0.001$ & 0.002 & 0.031 & 0.004 \\
\hline \multicolumn{6}{|l|}{ Diastolic pressure } \\
\hline$<80$ & 1.00 (reference) & 1.00 (reference) & 1.00 (reference) & 1.00 (reference) & 1.00 (reference) \\
\hline $80-89$ & $0.95(0.78-1.21)$ & $1.25(1.13-1.76)$ & $0.92(0.83-1.17)$ & $1.05(0.92-1.23)$ & $0.93(0.71-1.28)$ \\
\hline $90-99$ & $0.83(0.69-1.05)$ & $0.68(0.35-0.77)$ & $0.89(0.73-1.08)$ & $0.83(0.69-1.19)$ & $0.88(0.70-1.32)$ \\
\hline$>100$ & $0.72(0.61-0.85)$ & $0.95(0.82-1.13)$ & $0.83(0.72-0.99)$ & $0.35(0.17-0.92)$ & $0.69(0.38-1.29)$ \\
\hline per $10 \mathrm{mmHg}$ & $0.92(0.85-1.04)$ & $0.99(0.87-1.20)$ & $0.92(0.84-1.05)$ & $0.78(069-0.92)$ & $0.89(0.71-1.16)$ \\
\hline $\mathrm{P}$ for linear association & 0.038 & 0.256 & 0.142 & 0.179 & 0.335 \\
\hline \multicolumn{6}{|l|}{ Pulse pressure } \\
\hline$<45$ & 1.00 (reference) & 1.00 (reference) & 1.00 (reference) & 1.00 (reference) & 1.00 (reference) \\
\hline $45-54$ & $0.92(0.8 \mathrm{I}-1.05)$ & $0.59(0.47-0.78)$ & $1.12(0.93-1.38)$ & $0.89(0.68-1.07)$ & $1.21(1.06-1.47)$ \\
\hline $55-65$ & $0.71(0.60-0.88)$ & $0.51(0.39-0.67)$ & $0.79(0.68-0.98)$ & $0.53(0.4 \mathrm{I}-0.72)$ & $1.10(0.97-1.25)$ \\
\hline$>65$ & $0.65(0.48-0.79)$ & $0.39(0.26-0.59)$ & $0.70(0.61-0.79)$ & $0.70(0.49-1.12)$ & $0.78(0.71-0.95)$ \\
\hline Per $10 \mathrm{mmHg}$ & $0.82(0.78-0.87)$ & $0.71(0.60-0.83)$ & $0.82(0.69-0.94)$ & $0.81(0.7 I-0.98)$ & $0.82(0.74-0.99)$ \\
\hline $\mathrm{P}$ for linear association & $<0.001$ & $<0.001$ & $<0.001$ & 0.014 & 0.041 \\
\hline
\end{tabular}


primary headache. To be specific, migraine is a chronic and debilitating neurological disorder with a complex sensory dysfunction. ${ }^{17,18}$ Although its pathophysiological mechanisms remain unclear, some evidence showed that calcitonin generelated peptide (CGRP), a predominant neurotransmitter in capsaicin-sensitive sensory nerves, was involved in migraine. ${ }^{19}$ Migraine patients had a higher basal CGRP concentration in blood, and its intravenous administration could result in a migraine attack. ${ }^{20,21}$ Studies have shown that the decrease of CGRP synthesis and release would lead to the elevation of $\mathrm{BP}^{22}$ And the increase of CGRP or the enhanced vascular sensitivity response to CGRP could exert a protective effect on the occurrence of hypertension. ${ }^{23-25}$ Those mechanisms could be helpful for the interpretation of our results.

Moreover, TTH is also a primary headache affected approximately $46 \%$ global adults, which is characterized by a bilateral, non-throbbing headache with mild to moderate intensity. ${ }^{26}$ Animal studies have shown that activation of nitric oxide synthase (NOS) was involved in the sensitization of pain pathways, and the generation of nitric oxide (NO) and that NOS inhibitors could decrease the persistent pain. ${ }^{27}$ And studies have shown that the inhibition of NOS results in the significant elevation of BP in both humans and experimental animals. Furthermore, nitric oxide (NO), a molecule regulating the cerebral and extra cerebral cranial blood flow and arterial diameters, also play a significant role in migraine, TTH and cluster headache. ${ }^{24}$ Sensitization of pain pathways in the spinal cord may be caused by the generation of NO. ${ }^{25}$ These mechanisms also suggested that the pain symptoms may be negatively associated with BP.

Many factors contribute to the occurrence of headache, including caffeine, smoking, alcohol, and hormonal changes. ${ }^{28}$ A more detailed discussion of this study was the relationship between BP and headache in postmenopausal women. Studies have shown that some postmenopausal women were reported to experience a worsening of the migraine attacks, but most of them would relieve during this time. ${ }^{29}$ And the HRT has a different effect on migraine. Specially, most females who accepted HRT showed an improvement or complete remission of their headache, but some women reported that there is no change or worsening after taking HRT. ${ }^{30}$ However, for TTH, whether it is triggered by menses should be further evaluated. In this study, 125 (7.9\%) females developed the migraine and 137 (8.7\%) females developed the TTH in the follow-up, which demonstrated a not mere phenomenon that postmenopausal women had migraine and
TTH. And our study investigate the relationship between BP and headache among these people after controlling the several related factors, such as smoking status, age, caffeine consumption.

There were some limitations to the present study. Firstly, it was a single-center study and the sample size was relatively small. Multi-center prospective studies are needed to validate our results. Secondly, although the data were adjusted for confounding factors, other potential factors, such as environmental factors and genetic factors, ${ }^{31,32}$ were not included in this study. More patients need to be recruited for a more comprehensive evaluation. Thirdly, the effect of the HRT on BP should be investigated in future studies, which may extend this research.

\section{Conclusion}

In this prospective hospital-based study, we found negative associations of SBP and PP with new occurrence of headache, especially migraine and $\mathrm{TTH}$, but there is no relationship between DBP and the subsequent development of headache.

\section{Funding}

There is no funding to report.

\section{Disclosure}

The authors report no conflicts of interest in this work.

\section{References}

1. Negro A, Delaruelle Z, Ivanova TA, et al. European Headache Federation School of Advanced S. Headache Pregnancy. 2017;18 (1): 106 .

2. Collaborators GBDN. Global, regional, and national burden of neurological disorders, 1990-2016: a systematic analysis for the Global Burden of Disease Study 2016. Lancet Neurology. 2019;18 (5):459-480. doi:10.1016/S1474-4422(18)30499-X

3. Martelletti P, Steiner TJ, Bertolote JM, Dua T, Saraceno B. The definitive position of headache among the major public health challenges. An End to the Slippery Slopedisregard j Headache Pain. 2007;8(3):149-151.

4. Jackson JL, Mancuso JM, Nickoloff S, Bernstein R, Kay C. Tricyclic and Tetracyclic Antidepressants for the Prevention of Frequent Episodic or Chronic Tension-Type Headache in Adults: a Systematic Review and Meta-Analysis. J Gen Intern Med. 2017;32 (12):1351-1358. doi:10.1007/s11606-017-4121-z

5. Finocchi C, Sassos D. Headache and arterial hypertension. Neurol Sci. 2017;38(Suppl 1):67-72. doi:10.1007/s10072-017-2893-x

6. Friedman BW, Mistry B, West JR, Wollowitz A. The association between headache and elevated blood pressure among patients presenting to an ED. Am J Emerg Med. 2014;32(9):976-981. doi:10.1016/ j.ajem.2014.05.017

7. Tronvik E, Stovner LJ, Hagen K, Holmen J, Zwart JA. High pulse pressure protects against headache: prospective and cross-sectional data (HUNT study). Neurology. 2008;70(16):1329-1336. doi:10.1212/01.wnl.0000309222.79376.57 
8. Gus M, Fuchs FD, Pimentel M, Rosa D, Melo AG, Moreira LB. Behavior of ambulatory blood pressure surrounding episodes of headache in mildly hypertensive patients. Arch Intern Med. 2001;161 (2):252-255. doi:10.1001/archinte.161.2.252

9. Ornello R, Caponnetto V, Frattale I, Sacco S. Patterns of Migraine in Postmenopausal Women: a Systematic Review. Neuropsychiatr Dis Treat. 2021;17:859-871. doi:10.2147/NDT.S285863

10. Aegidius KL, Zwart JA, Hagen K, Schei B, Stovner LJ. Hormone replacement therapy and headache prevalence in postmenopausal women. Head-HUNT Study Eur Journal of neurology. 2007;14 (1):73-78. doi:10.1111/j.1468-1331.2006.01557.x

11. Headache Classification Committee of the International Headache S. The International Classification of Headache Disorders, 3rd edition (beta version). Cephalalgia. 2013;33(9):629-808. doi:10.1177/ 0333102413485658

12. Olesen J, Lipton RB. Migraine classification and diagnosis. Int Headache Soc Criteria Neurology. 1994;44(6 Suppl 4):S6-10.

13. Middeke M, Lemmer B, Schaaf B, Eckes L. Prevalence of hypertension-attributed symptoms in routine clinical practice: a general practitioners-based study. J Hum Hypertens. 2008;22 (4):252-258. doi:10.1038/sj.jhh.1002305

14. Bigal ME, Kurth T, Santanello N, et al. Migraine and cardiovascular disease: a population-based study. Neurology. 2010;74(8):628-635. doi:10.1212/WNL.0b013e3181d0cc8b

15. Scher AI, Terwindt GM, Picavet HS, Verschuren WM, Ferrari MD, Launer LJ. Cardiovascular risk factors and migraine: the GEM population-based study. Neurology. 2005;64(4):614-620. doi:10.1212/01.WNL.0000151857.43225.49

16. Gudmundsson LS, Thorgeirsson G, Sigfusson N, Sigvaldason H, Johannsson M. Migraine patients have lower systolic but higher diastolic blood pressure compared with controls in a population-based study of 21,537 subjects. Reykjavik Study Cephalalgia. 2006;26(4):436-444. doi:10.1111/j.14682982.2005.01057.x

17. Urits I, Clark G, An D, et al. An Evidence-Based Review of Fremanezumab for the Treatment of Migraine. Pain Therapy. 2020;9(1):195-215. doi:10.1007/s40122-020-00159-3

18. Sprenger T, Goadsby PJ. Migraine pathogenesis and state of pharmacological treatment options. BMC Med. 2009;7:71. doi:10.1186/17417015-7-71

19. Edvinsson L, Haanes KA, Warfvinge K, Krause DN. CGRP as the target of new migraine therapies - successful translation from bench to clinic. Nat Rev Neurol. 2018;14(6):338-350. doi:10.1038/s41582018-0003-1
20. Edvinsson L. Blockade of CGRP receptors in the intracranial vasculature: a new target in the treatment of headache. Cephalalgia. 2004;24(8):611-622. doi:10.1111/j.1468-2982.2003.00719.x

21. Juhasz G, Zsombok T, Modos EA, et al. NO-induced migraine attack: strong increase in plasma calcitonin gene-related peptide (CGRP) concentration and negative correlation with platelet serotonin release. Pain. 2003;106(3):461-470. doi:10.1016/j.pain.2003.09.008

22. Deng PY, Li YJ. Calcitonin gene-related peptide and hypertension. Peptides. 2005;26(9):1676-1685. doi:10.1016/j.peptides.2005.02.002

23. Li J, Kaminski NE, Wang DH. Anandamide-induced depressor effect in spontaneously hypertensive rats: role of the vanilloid receptor. Hypertension. 2003;41(3 Pt 2):757-762. doi:10.1161/01. HYP.0000051641.58674.F7

24. Olesen J. The role of nitric oxide (NO) in migraine, tension-type headache and cluster headache. Pharmacol Ther. 2008;120 (2):157-171. doi:10.1016/j.pharmthera.2008.08.003

25. Wu J, Fang L, Lin Q, Willis WD. Fos expression is induced by increased nitric oxide release in rat spinal cord dorsal horn. Neuroscience. 2000;96(2):351-357. doi:10.1016/S0306-4522(99) 00534-5

26. Medical Advisory S. Neuroimaging for the evaluation of chronic headaches: an evidence-based analysis. Ont Health Technol Assess Ser. 2010;10(26):1-57.

27. Ashina S, Bendtsen L, Ashina M. Pathophysiology of tension-type headache. Curr Pain Headache Rep. 2005;9(6):415-422. doi:10.1007/s11916-005-0021-8

28. Peroutka SJ. What turns on a migraine? A systematic review of migraine precipitating factors. Curr Pain Headache Rep. 2014;18 (10):454. doi:10.1007/s11916-014-0454-z

29. Wang SJ, Fuh JL, Lu SR, Juang KD, Wang PH. Migraine prevalence during menopausal transition. Headache. 2003;43(5):470-478. doi:10.1046/j.1526-4610.2003.03092.x

30. Mueller L. Predictability of exogenous hormone effect on subgroups of migraineurs. Headache. 2000;40(3):189-193. doi:10.1046/j.15264610.2000.00027.x

31. Kroner-Herwig B, Gassmann J. Headache disorders in children and adolescents: their association with psychological, behavioral, and socio-environmental factors. Headache. 2012;52(9):1387-1401. doi:10.1111/j.1526-4610.2012.02210.x

32. Russell MB. Genetics in primary headaches. $J$ Headache Pain. 2007;8(3):190-195. doi:10.1007/s10194-007-0389-4
International Journal of General Medicine

\section{Publish your work in this journal}

The International Journal of General Medicine is an international, peer-reviewed open-access journal that focuses on general and internal medicine, pathogenesis, epidemiology, diagnosis, monitoring and treatment protocols. The journal is characterized by the rapid reporting of reviews, original research and clinical studies across all disease areas. The manuscript management system is completely online and includes a very quick and fair peer-review system, which is all easy to use. Visit http://www.dovepress.com/ testimonials.php to read real quotes from published authors. 